\title{
Prenatal androgen exposure causes a sexually dimorphic transgenerational increase in offspring susceptibility to anxiety disorders
}

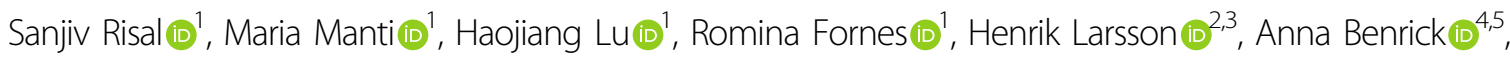 \\ Qiaolin Deng (1)', Carolyn E. Cesta $\mathbb{1}^{6}$, Mina A. Rosenqvist (10 ${ }^{3}$ and Elisabet Stener-Victorin (1)
}

\begin{abstract}
If and how obesity and elevated androgens in women with polycystic ovary syndrome (PCOS) affect their offspring's psychiatric health is unclear. Using data from Swedish population health registers, we showed that daughters of mothers with PCOS have a $78 \%$ increased risk of being diagnosed with anxiety disorders. We next generated a PCOSlike mouse $\left(F_{0}\right)$ model induced by androgen exposure during late gestation, with or without diet-induced maternal obesity, and showed that the first generation $\left(F_{1}\right)$ female offspring develop anxiety-like behavior, which is transgenerationally transmitted through the female germline into the third generation of female offspring $\left(F_{3}\right)$ in the androgenized lineage. In contrast, following the male germline, $\mathrm{F}_{3}$ male offspring $\left(\mathrm{mF}_{3}\right)$ displayed anxiety-like behavior in the androgenized and the obese lineages. Using a targeted approach to search for molecular targets within the amygdala, we identified five differentially expressed genes involved in anxiety-like behavior in $F_{3}$ females in the

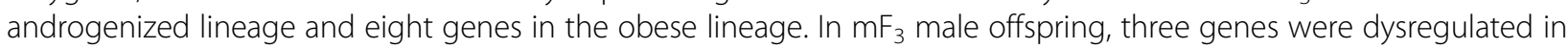
the obese lineage but none in the androgenized lineage. Finally, we performed in vitro fertilization (IVF) using a PCOS mouse model of continuous androgen exposure. We showed that the IVF generated $F_{1}$ and $F_{2}$ offspring in the female germline did not develop anxiety-like behavior, while the $F_{2}$ male offspring $\left(\mathrm{mF}_{2}\right)$ in the male germline did. Our findings provide evidence that elevated maternal androgens in PCOS and maternal obesity may underlie the risk of a transgenerational transmission of anxiety disorders in children of women with PCOS.
\end{abstract}

\section{Introduction}

Polycystic ovary syndrome (PCOS) is recognized as a heterogeneous disorder affecting over $15 \%$ of women in the general population and over $25 \%$ of women with obesity $^{1,2}$. Over $60 \%$ of women with PCOS are diagnosed with at least one psychiatric disorder ${ }^{3,4}$. Elevated circulating androgens is the most prominent feature of PCOS, which persists throughout reproductive life and even after

\footnotetext{
Correspondence: Elisabet Stener-Victorin (elisabet.stener-victorin@ki.se) ${ }^{1}$ Department of Physiology and Pharmacology, Karolinska Institutet, Stockholm, Sweden

${ }^{2}$ Department of Medical Epidemiology and Biostatistics, Karolinska Institutet, Stockholm, Sweden

Full list of author information is available at the end of the article These authors jointly supervised this work: Carolyn E. Cesta, Mina A. Rosenqvist, Elisabet Stener-Victorin
}

menopause ${ }^{5,6}$, and reproductive, metabolic and psychiatric dysfunction are all positively correlated with hyperandrogenaemia $^{4-7}$.

Prenatal androgen exposure has been proposed to have a potential causal influence on the development of neuropsychiatric disorders in children born to women with PCOS in a Swedish register-based studies ${ }^{8-10}$. Specifically, an increase in the risk of attention-deficit/hyperactive disorder (ADHD) and autism spectrum disorder (ASD) in daughters, and to a lesser extent in sons of women with PCOS has been found even when accounting for genetic factors ${ }^{8}$. Similarly, a recent study has reported that children born to women with PCOS had higher risk for childhood anxiety diagnoses ${ }^{11}$, and maternal PCOS, independently, and jointly with maternal obesity, has been 
shown to be associated with increased risks for almost all groups of psychiatric and mild neurodevelopmental disorders in offspring ${ }^{12}$. Whether the risk is different for daughters and sons remains to be investigated.

Prenatal androgenized rodent studies have demonstrated that maternal androgen excess may underpin the risk of developing anxiety disorders in female offspring and to a lesser extent in male offspring ${ }^{13,14}$, whereas dietinduced maternal obesity increases anxiety-like behavior only in the male offspring ${ }^{14}$. Using the prenatal androgenized mouse model combined with a diet-induced maternal obesity model we recently demonstrated that elevated maternal androgens, and to lesser extent maternal obesity, reprogram the fetus and induce a transgenerational increase in female offspring susceptibility to develop a PCOS-like phenotype ${ }^{15}$. To ascertain transgenerational inheritance, the phenotypic changes must be manifested in $F_{3}$ offspring because $F_{1}$ fetuses and the already developing germline (that will give rise to the $F_{2}$ ) are also directly exposed ${ }^{15}$. Thus, whether elevated maternal androgens potentiate transgenerational susceptibility to anxiety-like behavior in adult female and male offspring has not been explored.

\section{Results}

\section{Daughters of women with PCOS are diagnosed with anxiety disorders}

We conducted a Swedish nationwide register-based cohort study to assess if daughters of women with PCOS have a higher risk of being diagnosed with anxiety disorders. By linking the Swedish Medical Birth Register (MBR) and the National Patient Register (NPR), a total of 102,466 children were identified, of which 8864 (8.7\%) were born to mothers with a diagnosis of PCOS. Maternal and child characteristics are reported in Table S1. Overall, $1.32 \%$ of children $(n=117)$ born to a mother with PCOS had a diagnosis of anxiety made in inpatient or outpatient hospital-based specialized care, compared to $0.97 \%$ ( $n=$ 912) of children born to mothers without a PCOS diagnosis. The mean age (and standard deviation) of first anxiety diagnosis for all children was $13.5 \pm 3.0$ years, and $12.4 \pm 3.1$ years and $14.1 \pm 2.8$ years for boys and girls, respectively. Table S2 shows the crude and adjusted hazard ratios (HR). Children born to mothers with PCOS had an increased risk of being diagnosed with anxiety (adjusted model, HR $=1.49$ 95\% CI 1.16-1.92). When adjusting the models for parental anxiety diagnoses, instead of any psychiatric diagnosis, the estimates remained similar (data not shown). When stratified by child's sex (Table S2), daughters born to women with PCOS $(n=4290)$ had a $78 \%$ increased risk of being diagnosed with anxiety compared to daughters born to women without PCOS (adjusted model, HR $=1.78$ 95\% CI 1.19-2.67). In contrast, sons born to women with
Risk of anxiety diagnosis in children of mothers with PCOS
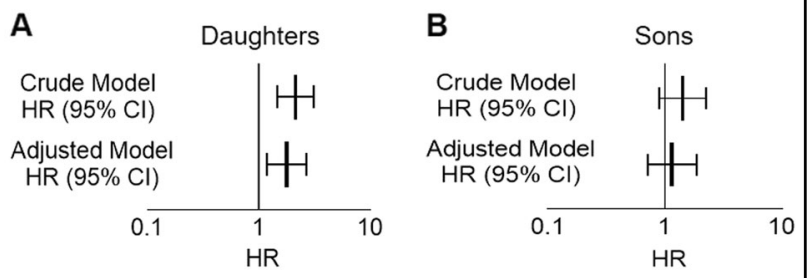

Fig. 1 Risk of having an anxiety in daughters of women with PCOS. A, B Crude and adjusted hazard ratios (HRs) and 95\% confidence intervals (Cls) for the association between maternal PCOS and daughter's diagnosis of anxiety in the Swedish nation-wide register-based study. Adjusted model is adjusted for maternal age, maternal country of birth, maternal education, maternal and paternal psychiatric history, and year of birth of child.

PCOS $(n=4574)$ had no increase in risk for a diagnosis of anxiety (Adjusted HR $=1.15$ 95\% CI 0.71-1.86) (Fig. 1a). The sensitivity analysis in the subset of the population with BMI information produced a similar pattern of estimates as the main analysis however the estimates were attenuated with wider confidence intervals (Table S3).

\section{Prenatal androgen exposure and maternal obesity cause transgenerational anxiety-like behavior}

Next, we aimed to elucidate whether female and male offspring $\left(\mathrm{F}_{1}\right)$ of maternal androgen exposed mothers $\left(\mathrm{F}_{0}\right)$ fed either control diet $(\mathrm{CD})$ or high-fat high sugar diet (HFHS) are susceptible to develop anxiety-like behavioral traits in adulthood, and if the anxiety-like behavior phenotype is transmitted to the second $\left(\mathrm{F}_{2}\right)$ and thirdgeneration $\left(\mathrm{F}_{3}\right)$ i.e., transgenerational (Fig. $2 \mathrm{~A}$ and Table S4). $F_{1}$ female offspring in the androgenized, the obese, and in the combined obese and androgenized lineages spent less time in the open arms and more time in the closed arms of the elevated plus maze (Fig. 2B), indicating that prenatal androgen exposure combined with maternal obesity aggravates anxiety-like behavior. There was a main effect of the androgenized lineage and the obese lineage in the elevated plus maze [open arms, androgenized: $F_{1,78}=11.5, P=0.001$ and obese: $F_{1,78}=19.28, P<$ 0.0001], [closed arms, androgenized: $F_{1,78}=4.83, P=$ 0.006 and obese: $\left.F_{1,78}=16.27, P=0.0001\right]$. Further, there was an interaction between the androgenized and the obese lineages in the open field, wherein the $F_{1}$ female offspring in the androgenized lineage spent less time in the center [center: $F_{1,78}=4.86, P=0.030$ ] and more time in the periphery [periphery: $F_{1,78}=4.82, P=0.031$ ], while the $F_{1}$ females in the combined lineages spent more time in the center and less time in the periphery of the open field (Fig. 2B). Furthermore, in line with human data, $F_{1}$ male offspring did not present any anxiety-like behavioral changes (Fig. 3A). 


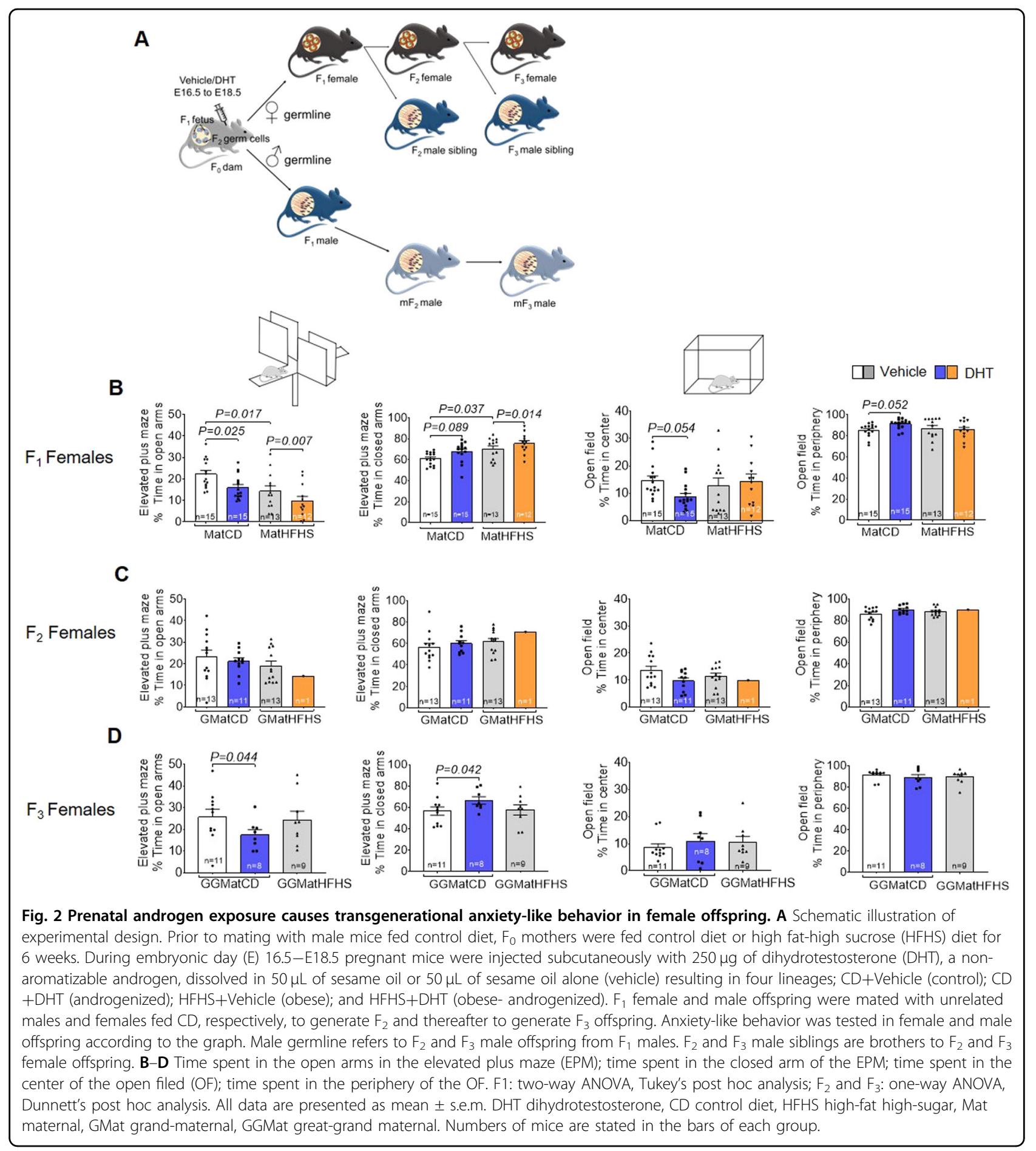

Exploring the transgenerational transmission of anxietylike behavior, we show that $F_{3}$ female offspring in the androgenized lineage spent less time in the open arms and more time in the closed arms of the elevated plus maze, with no differences in the open field (Fig. 2D). No changes were observed in the $\mathrm{F}_{2}$ female offspring (Fig. 2C) and in the male $F_{2}$ and $F_{3}$ offspring, i.e., siblings (Supplementary
Fig. 1A, B). The male germline, $\mathrm{mF}_{3}$ male offspring in the androgenized lineage and the $\mathrm{mF}_{3}$ male offspring in the obese lineage spent less time in the open arms, and more time in the closed arms of the elevated plus maze (Fig. 3C) but no difference in time spent in center and periphery of the open field. There was an interaction between the androgenized and the obese lineages in the elevated plus 


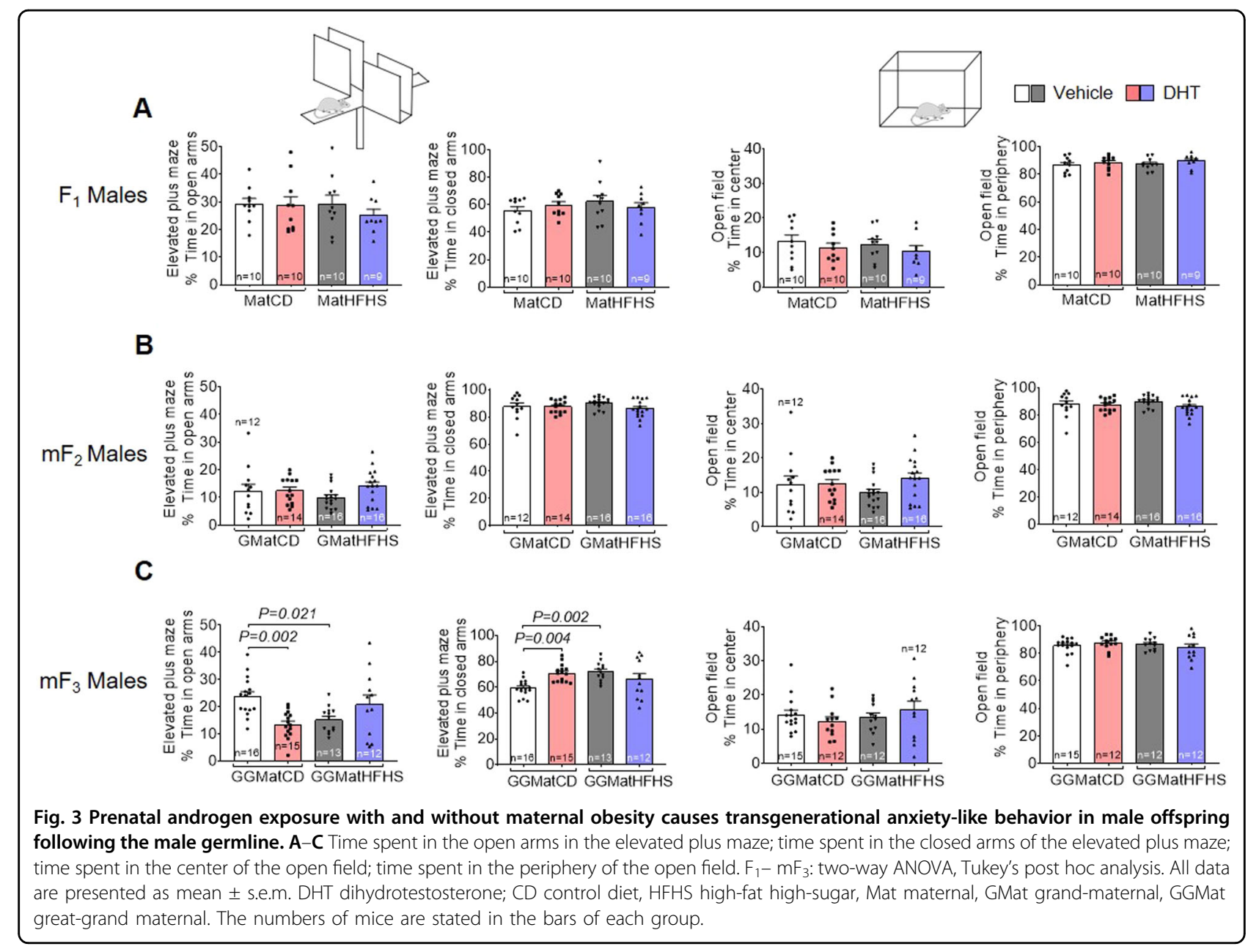

maze in $\mathrm{mF}_{3}$ male offspring [open arms: $F_{1,52}=14.93$, $P=0.0003$ ] and [closed arms: $F_{1,52}=12.35, P=0.0009$ ]. No changes were observed in the $\mathrm{mF}_{2}$ male offspring (Fig. 3B). These findings suggest that the anxiety-like behavioral phenotype can be transmitted across generations in female offspring from androgenized $\mathrm{F}_{0}$ mothers, but not in the male siblings. Further, $\mathrm{mF}_{3}$ male offspring following the male germline displays an anxiety-like behavior either due to maternal obesity or androgen exposure in $F_{0}$ mothers.

\section{Prenatal androgen exposure and obesity resulted in altered amygdala gene expression}

As there is a strong indication that prenatal androgen exposure and obesity cause a sexually dimorphic anxietylike behavior across generations, we performed targeted gene expression analyses within the amygdala of the $\mathrm{F}_{3}$ female and the $\mathrm{mF}_{3}$ male offspring using a low-density TaqMan array (Fig. 4A and Table S5). Based on our previous single-cell RNA sequencing data of mouse MII oocytes $^{15}$, we selected differentially expressed genes (DEGs) across generations related to anxiety in the androgenized and in the obese lineages (Fig. 4B). In addition, known genes involved in anxiety were selected for analyses ${ }^{14,16-18}$ (Table S5). Several genes related to anxiety showed differential expression in the amygdala in the obese and androgenized lineages of $\mathrm{F}_{3}$ female and $\mathrm{mF}_{3}$ male offspring. Specifically, in the androgenized and the obese lineages of $F_{3}$ female offspring, we found downregulated expression of the calcium/calmodulindependent protein kinase II inhibitor 1 (Camk2n1), and the calcium voltage-gated channel auxiliary subunit alpha 2 delta 1 (Cacna2d1) ${ }^{19}$ involved in the regulation of $\mathrm{Ca}^{2+}$ influx through voltage-gated calcium channels; the solute carrier family 17 member 7 (Slc17a7) $)^{20}$ involved in glutamate uptake into synaptic vesicles; the dopamine beta-hydroxylase $(D b h)^{21}$ catalyzing the conversion of dopamine to norepinephrine; and the BTG anti-proliferation factor $2(B \operatorname{tg} 2)^{22,23}$ involved in neurite outgrowth (Fig. 4C-G). In addition, only in the obese lineage of $\mathrm{F}_{3}$ female offspring we found an upregulation of the Forkhead box P2 (Foxp2), a transcription factor involved in brain development ${ }^{24}$; the TIA1 cytotoxic granule associated RNA binding protein-like 1 (Tial1), 


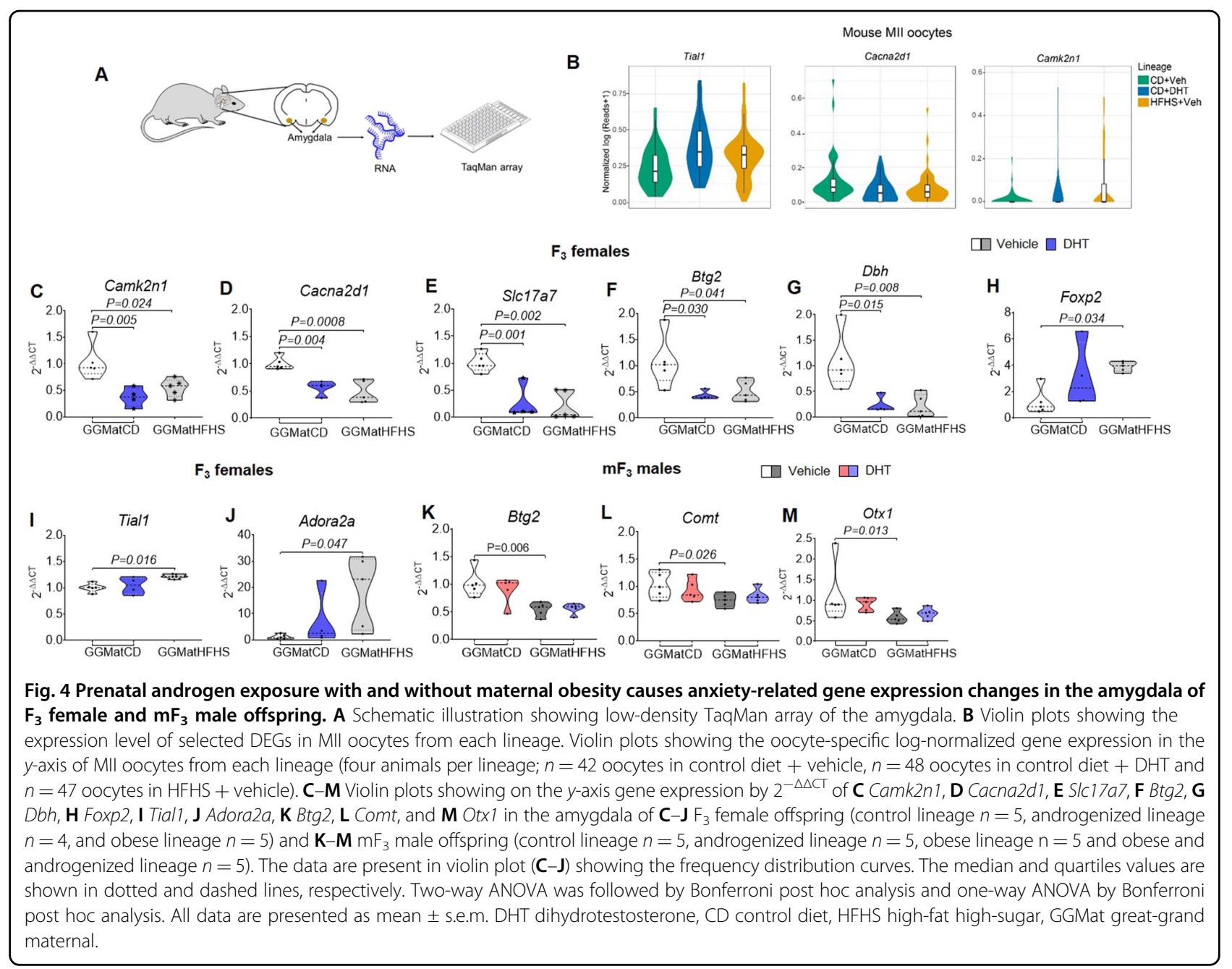

involved in synaptic plasticity and the processing of fear memory ${ }^{25}$; and adenosine A2a receptor (Adora2a), which influences the fine-tuning of neurotransmitters such as dopamine ${ }^{26}$ (Fig. $4 \mathrm{H}-\mathrm{J}$ ). In $\mathrm{mF}_{3}$ male offspring, only the obese lineage showed downregulated expression of the Btg2, the Catechol-O-Methyltransferase $(\mathrm{Com} t)^{27}$ involved in catecholamine metabolism, and the Orthodenticle homeobox $1(\operatorname{Otx} 1)^{28}$ involved in brain development (Fig. 4K-M).

\section{Germ cells versus in utero environment in the transmission of anxiety-like phenotype in offspring}

Finally, to delineate germ cell factors from the in utero environment in the transmission of anxiety-like phenotype in offspring in PCOS, we used the prepubertal mouse model of $\mathrm{PCOS}^{29}$ combined with in vitro fertilization (IVF) and embryo transfer into surrogate mothers (Fig. 5A). Neither $F_{0}$ donors exposed continuously to DHT from puberty until superovulation for oocyte donation (Fig. 5B), nor did the $F_{1}$ female and male offspring display an anxiety-like behavior in the elevated-plus maze and open field tests (Fig. 5C, D). Further, $\mathrm{F}_{2}$ female and male offspring in the female germline did not display signs of anxiety-like behavior (Fig. 5E, F). However, following the male germline, the $F_{2}$ male but not the $F_{2}$ female offspring in the DHT lineage displayed an anxiety-like behavior with more time spent in the periphery of the open field (Fig. 5G, H).

Thus, these two sets of experiments in the PNA and prepubertal mouse models of PCOS suggest that the transgenerational transmission of anxiety-like behavior in females following the female germline is likely due to an aberrant health of somatic cells, whereas the transgenerational transmission of anxiety-like behavior in the $\mathrm{F}_{2}$ male offspring following the male germline is likely mediated via modifications in the germ cells. The observed male germline effects on anxiety-like behavior is in line with previous studies demonstrating transmission of the epigenetic mark through the male gametes to their offspring $^{30,31}$. 


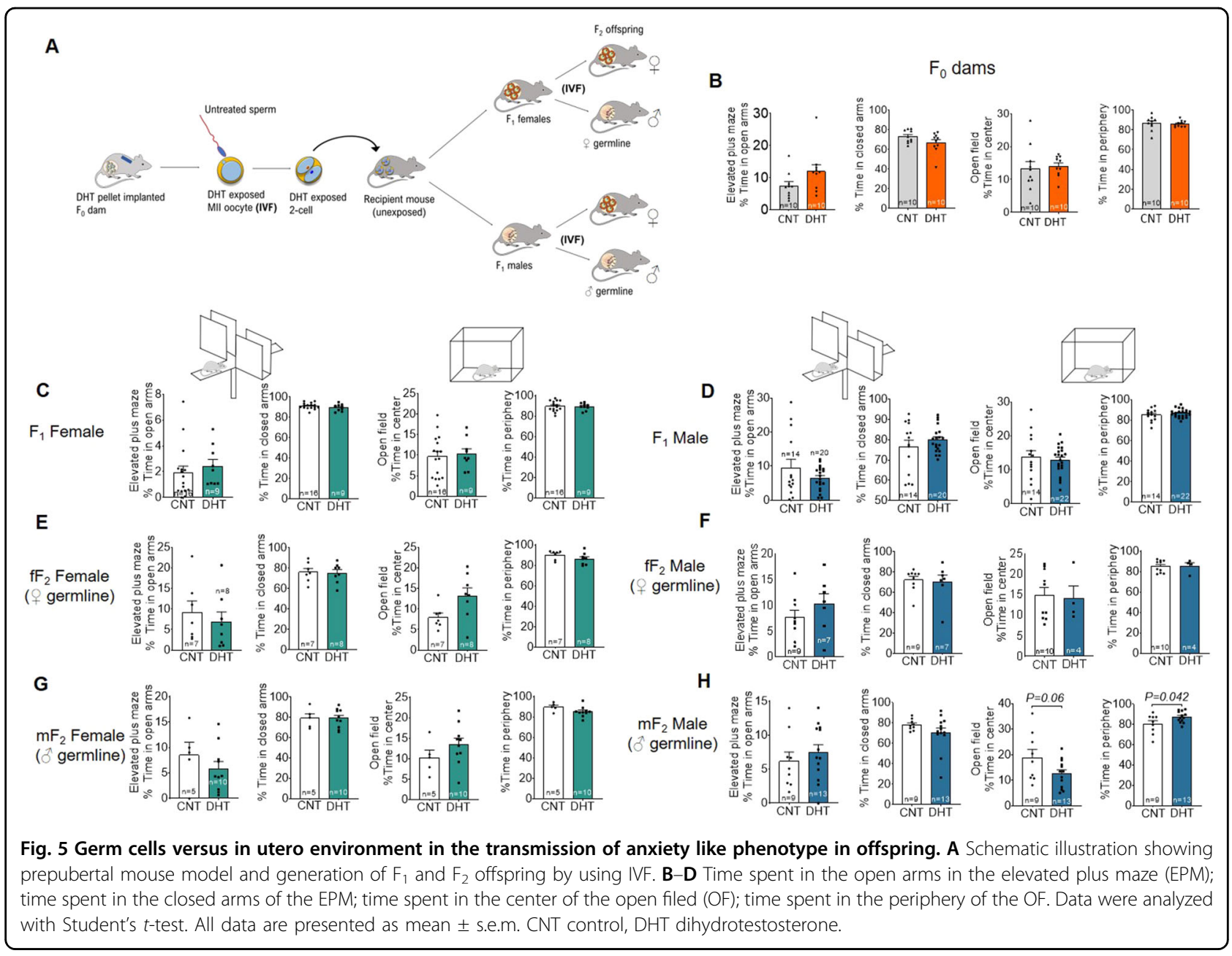

\section{Discussion}

Here, we studied how anxiety disorders are propagated across generations due to maternal PCOS conditions in both human and mouse studies. Our findings showed that daughters, but not sons of women with PCOS are at a 78\% higher risk of being diagnosed with anxiety disorders compared with children born to women without PCOS. In a PCOS-like mouse model, prenatal exposure to androgens caused an anxiety-like behavior only in the $F_{1}$ female offspring, which was transgenerationally inherited to the $\mathrm{F}_{3}$ female offspring, but not to the male sibling offspring. Maternal obesity led to an increased anxiety-like behavior in $F_{1}$ female offspring, but such effects were not observed in $F_{3}$ offspring via the female germline. In the male germline, $\mathrm{mF}_{3}$ males developed anxiety-like behavior in the androgenized and in the obese lineages. Within the amygdala of $\mathrm{F}_{3}$ female offspring, five genes (Camk2n1, Cacna2d1, Slc17a7, Btg2, and Dbh) were downregulated in the androgenized and obese lineages and three genes (Foxp2, Tial1, and Adora2a) were upregulated only in the obese lineage. Lastly, Btg2, Comt, and Otx1 were downregulated within the amygdala of the $\mathrm{mF}_{3}$ male offspring in the obese lineage.

Prenatal androgen exposure may cause permanent reprogramming of fetal neural systems thereby increasing the risk of neuropsychiatric disorders ${ }^{8,32}$. In our human and mouse studies, maternal PCOS and in utero androgen exposure, respectively, led to increased anxiety behaviors in $F_{1}$ female offspring, but not in $F_{1}$ male offspring. The mechanism leading to this sexual dimorphic response in behavior is not fully understood, but different hypotheses have been speculated. During the embryonic development, female fetuses are exposed to lower levels of androgens, whereas male fetuses encounter higher levels of androgens from 8th to 24th weeks of gestation as their developing testes also produce androgens ${ }^{33}$. Pregnant women with PCOS have higher levels of androgens throughout pregnancy compared to pregnant women without $\mathrm{PCOS}^{34-40}$. Therefore, while both male and female fetuses of women with PCOS encounter higher prenatal androgen levels, it can be hypothesized that the effects on brain development and behavior later in life are 
stronger in females. Similarly to these observations and our findings, previous studies in animal models of PCOS have shown a sexually dimorphic anxiety-like behavior, with increased anxiety-like behavior in female offspring, which was further supported by sex-specific gene expression changes in the amygdala ${ }^{13,14,41}$. Intrinsic epigenetic differences between the daughters and sons of women with PCOS leading to differential trajectories of DNA methylation in the brain development could also be involved in the observed behavioral sex differences ${ }^{42,43}$. However, studies that investigate epigenetics in the neurodevelopment in PCOS are lacking.

There is accumulating evidence that an adverse in utero environment can influence not only the first generation of offspring but also the subsequent generations ${ }^{15}$. Data from a few epidemiological studies along with studies in animal models have increased our understanding of the effects, and the mechanisms by which changes in the maternal environment during pregnancy can affect the health of children and grandchildren ${ }^{44,45}$. In the current study, we investigated how a maternal environment with androgen excess and obesity affects the behavior of three subsequent generations. Interestingly, we demonstrated that maternal androgen excess had a stronger impact on the transgenerational transmission of anxiety-like behavior in the offspring, rather than maternal obesity. The existing data provide support for transmission of behavioral changes, including anxiety disorders and autism spectrum disorders, in the first generation ${ }^{9,46-48}$, but the evidence is limited for subsequent generations. Other adverse maternal stimuli, such as maternal exposure to glucocorticoids, are shown to increase stress responses and anxiety disorders in the 1st and 2nd generation of offspring, with female offspring being more affected ${ }^{49,50}$.

The transgenerational effects of an adverse maternal environment have been studied more thoroughly than the effects of an adverse paternal environment in the offspring. However, an increasing number of animal studies assess the paternal transmission of effects, providing more accurate information about the involved mechanisms, as many confounding factors are eliminated ${ }^{45,51}$. Paternal transmission of phenotypes is inherited through the genetic and epigenetic material in the sperm, while the maternal transmission may be mediated by germ cells and/or the maternal environment during gestation and lactation. Here, we studied both maternal and paternal transmission of anxiety-like behavior. With regard to the paternal transmission, we showed that the $F_{1}$ male offspring exposed to maternal androgens and/or obesity in utero did not develop behavioral changes, but they did transmit an anxiety-like behavior in the third generation of male offspring $\left(\mathrm{mF}_{3}\right)$ in the androgenized and the obese lineages, respectively. These findings suggest that the observed changes in the $\mathrm{mF}_{3}$ offspring may be transmitted through germline inheritance. In the IVF experiment, we showed that $\mathrm{mF}_{2}$ male offspring developed an anxietylike behavior, likely supporting the transmission of anxiety-like behavior through the germ cells. Future experiments investigating epigenetic changes in the sperm are warranted.

The amygdala consists of multiple interconnected nuclei that are involved in emotional processing and play a crucial role in the development of anxiety ${ }^{13,14,52}$. To define the molecular mechanisms underlying the observed transgenerational transmission of anxiety-like behavior in $\mathrm{F}_{3}$ female offspring and in $\mathrm{mF}_{3}$ male offspring, we analyzed target genes within their amygdala. We found a downregulated expression of genes involved in calcium homeostasis (Camk2n1, Cacna2d1), glutamate transport (Slc17a7), neurite growth regulator (Btg2), and dopamine metabolism $(D b h)$ in both the androgenized and obese lineage of $\mathrm{F}_{3}$ female offspring. It has been reported that CAMK2N1 expression is downregulated after androgen receptor (AR) activation in human prostate cancer cell lines following an auto-regulatory negative feedback loop ${ }^{53,54}$. We have also shown a dysregulated expression of Camk2n1 in the oocytes of the androgenized females. Thus, it can be speculated that Camk $2 n 1$ expression is dysregulated in multiple organs in our prenatally androgenized model due to changes in AR signaling. Calcium voltage-gated channels are important for neurotransmission, and genomic aberrations of the CACNA2D1 gene have been linked to autism spectrum disorder, epilepsy, and neuropsychiatric disorders ${ }^{19,55}$. Therefore, it can be surmised that prenatal androgenization of $F_{1}$ females resulted in reprogramming of the amygdala and led to dysregulation of genes involved in calcium signaling, among others, causing an anxiety-like behavior in the $F_{3}$ female offspring.

In the male germline, $\mathrm{mF}_{3}$ male offspring in the androgenized and obese lineages displayed an anxiety-like behavior. Among the tested genes, none was found dysregulated in the androgenized lineage, but Comt, Btg2, and Otx 1 were downregulated in the obese lineage. COMT is an enzyme involved in the inactivation of catecholamines, and it has been associated with a broad range of psychiatric phenotypes in human and animal studies $^{56}$. Interestingly, COMT val18met polymorphism has been associated with both anxiety disorders ${ }^{57}$ and abdominal obesity in men, findings that are in line with our data ${ }^{58}$. BTG2 has been shown to interact with AR, by repressing AR transcriptional activity ${ }^{57}$. Other mouse studies have shown downregulation of Btg2 in the medial prefrontal cortex of male mice with social behavior defi$\operatorname{cits}^{59}$, and upregulation in the neocortex of mice in response to acute immobilization stress ${ }^{60}$. Finally, Otx 1 is a transcription factor that is evidenced to play an important role in brain development ${ }^{61}$. Mice lacking 
Otx1 develop epilepsy and abnormalities in many brain regions $^{62}$.

Our recent publication shows that androgen is the main driver and likely reprograms germ cells and/or adversely affects the in utero environment, propagating a PCOS-like phenotype up to three generations. Here, we extend these findings and confirm that an anxiety-like phenotype is transgenerationally transmitted only in the androgenized lineage in females, whereas it is transmitted to the third generation in both the obese and androgenized lineages in $\mathrm{mF}_{3}$ male offspring. To dissect germ cell factors from the in utero environment in the transmission of anxiety-like phenotype in PCOS female and male offspring, we used the prepubertal mouse model of PCOS and combined it with IVF and embryo transfer to a healthy surrogate mother to generate $F_{1}$ and $F_{2}$ offspring. Intriguingly, the female offspring did not display anxiety-like behavior. On the other hand, $\mathrm{mF}_{2}$ male offspring in the androgenized lineage displayed anxiety-like behavior demonstrating that androgen exposed oocytes carry reprogrammed information and transmit it transgenerationally to the $\mathrm{mF}_{2}$ male offspring.

Although differences do exist, the circuitry and function of the amygdala are well-conserved across species ${ }^{63,64}$. Therefore, our rodent behavioral experiments and subsequent molecular analyses provide some clues to the potential pathways behind the increased risk for anxiety diagnoses found in the daughters of women with PCOS.

Two other studies have investigated anxiety in the children born to women with PCOS. Robinson et al. ${ }^{11}$ reported a $62 \%$ increased risk of a childhood anxiety diagnosis when compared to children born to women without PCOS. This study was conducted in a US population-based birth cohort, where both the PCOS status of the mother and any childhood anxiety diagnosis was reported by the mother when the children 7 or 8 years old. Chen et al. ${ }^{12}$ used a Finnish population-based cohort study and reported a 33\% increased risk (HR 1.33; 95\% CI 1.26-1.41) for an anxiety diagnosis in children born to women with PCOS, however they did not find a difference for male and female children. Comparatively, we report a slightly higher risk for anxiety overall, and specifically an increased risk for daughters born to women with PCOS.

The strength in our study lies in the large study size due to the use of health register data covering the entire population in Sweden. Additionally, data on diagnoses of both PCOS and anxiety were reported from hospitalbased specialist care to the registries, and we had a long follow-up time for the children. Nonetheless, there are some inherent limitations to observational register-based data. The prevalence of anxiety amongst the children in the study population was lower than previously reported $^{65}$, likely because we do not include diagnoses made in primary care, and possibly because the study population is younger than the estimated mean age at onset of anxiety $(21.3 \text { years })^{66}$. Further, it is well established that women with PCOS have increased rates of anxiety ${ }^{7}$. In our study, while we accounted for parental history of psychiatric disorders in the analysis, we were unable to investigate separately the role of genetic (i.e., related to anxiety and behavior) and environmental factors (e.g., prenatal androgen exposure, maternal stress) which could influence the risk of anxiety disorders in the children. The mothers with PCOS in the study had elevated BMI compared to mothers without PCOS. However, due to differential missingness of the BMI information, we avoided biasing our results by not including BMI in the main analysis models. Instead we conducted a complete case analysis, and despite the slight difference between the subsample and the full cohort, the estimates followed a similar pattern as in the main analysis albeit with attenuated HRs and wider confidence intervals due to the lower numbers. However, this strengthens the findings in the main analysis, as the association is found is not completely attributable to maternal BMI. Similarly, Chen et al. ${ }^{12}$ found that their results suggested a synergistic effect of PCOS and obesity on offspring anxiety. This agrees with the mouse model experiments where the influence of maternal obesity was explored. Further, due to the young age of the cohort, we were unable to assess any transgenerational effects of maternal PCOS on subsequent generations. The strength of our study is that we translate our preclinical mice data to real-life clinical data.

In summary, we here show that daughters of women with PCOS are more likely to develop anxiety disorders, but not sons. In a mouse model of PCOS, we revealed that maternal androgen excess causes transgenerational transmission of anxiety-like behavior in female offspring $\left(F_{1}\right.$ and $\left.F_{3}\right)$, but not in $F_{1}, F_{2}$, and $F_{3}$ male siblings. We further demonstrated that the first generation of unaffected male offspring exposed to maternal androgens and maternal obesity did also transmit an anxiety-like behavior in subsequent male generations $\left(\mathrm{mF}_{3}\right)$. These behavioral changes in the $\mathrm{F}_{3}$ female offspring and $\mathrm{mF}_{3}$ male offspring were accompanied by gene expression changes in the amygdala. Our findings provide evidence that elevated maternal androgens in PCOS accompanied by maternal obesity may underlie the risk of transgenerational transmission of anxiety disorders in daughters and sons of women with PCOS, an effect that can be mediated by epigenetic reprogramming.

\section{Materials and methods Ethical approvals}

The authors assert that all procedures contributing to this work comply with the ethical standards of the relevant national and institutional committees on human experimentation and with the Helsinki Declaration of 
1975 , as revised in 2008. The cohort study was approved by the regional ethical review board in Stockholm, Sweden (diary number 2013/862-31/5; 2016/1214-32). The requirement for informed consent was waived because the study was register-based, and the included individuals were not identifiable at any time.

Animal experiments were done in accordance with the legal requirements of the European Community (SJVFS 2017:40) and the directive 2010/63/EU of the European Parliament on the protection of animals used for scientific purposes and approved by the Stockholm Ethical Committee for Animal Research (Dnr. No. 10798-2017). Animal care and procedures were controlled by Comparative Medicine Biomedicum (KM-B), Karolinska Institutet, Stockholm, Sweden.

\section{Register-based cohort study population}

Using the unique personal identification number assigned to each individual in Sweden at birth or at immigration, several nationwide longitudinal registers containing health and sociodemographic data until December 31, 2013, were linked, including the Swedish Medical Birth Register (MBR), National Patient Register (NPR), Prescribed Drug Register (PDR), Total Population Register (TPR), Migration Register, and the Cause of Death Register (CDR).

Women who had delivered at least one child were identified through the MBR. The NIH diagnostic criteria for PCOS were established in 1990 followed by the Rotterdam criteria $2003^{67}$. Women with PCOS were identified by having at least one PCOS International Classification of Diseases (ICD) code (ICD-9: 256E; ICD-10: E28.2) recorded in the MBR or in the NPR after the age of 13 and between 1990 and 2013. Women with a concurrent diagnosed condition that could cause symptoms similar to PCOS were excluded to ensure specificity ${ }^{4,8}$. This yielded a total of 12,955 mothers with PCOS. Time of PCOS diagnosis (before or after pregnancy) was not taken into account since elevated testosterone levels have been reported to be present throughout life in women with $\mathrm{PCOS}^{5}$. Each mother with PCOS was then matched to upto ten comparison mothers without a PCOS diagnosis randomly selected from the general population on her birth year and county of residence within the year of diagnosis.

Children born from 1995 to 2007 to mothers with PCOS and matched unaffected mothers were identified. All children from each mother were included. Children were excluded if they were born outside of Sweden, adopted, stillborn or died on the day of birth, or had congenital malformations. This yielded a total of 8864 children born to women with PCOS and 89,431 children born to women without PCOS from the general population. The birth years of 1995 to 2007 were chosen so that the children had complete coverage in the outpatient register (which began in 2001) from the age-eligible for anxiety diagnoses (age 6). Children were followed until December 31, 2013, yielding an age range of 6-18.9 years at the end of follow-up.

\section{Outcome classification}

Diagnoses of anxiety recorded after the age of 6 were identified by ICD-10 codes F40.0, F40.1, F41.0, F41.1, and F43.

\section{Covariates}

Highest attained maternal education level (primary and secondary education, upper secondary education, postsecondary/post-graduate education) was used as a proxy for the child's socioeconomic status. Maternal and paternal lifetime history of psychiatric disorders was determined by any recorded psychiatric diagnosis in the NPR. Region of birth (Nordic/non-Nordic) was extracted from the TPR. The child's sex and year of birth were extracted from the MBR. Data on maternal body mass index (BMI) was available in the MBR, however, due to a high proportion of missing values (13\%) combined with data not missing at random (missingness was associated with the outcome) it was not included in the main analysis so as not to introduce bias.

\section{Animals}

The number of mice used for behavioral testing and for gene expression analyses is given in the figure legend and/ or text. Details of breeding of females and male sibling have previously been described ${ }^{15}$ and is detailed in Table S4. In brief, 21-days-old C57Bl/6J mice were obtained from Janvier Labs (Le Genest-Saint-Isle, France). All mice were maintained under a 12-h light/dark cycle and in a temperature-controlled room with ad libitum access to water and a diet. After one week of acclimatization, female mice were randomly divided into two groups and fed either 1) a CD (Research Diets, D12328) comprising 11\% fat, $73 \%$ carbohydrates [ $0 \%$ sucrose], and $16 \%$ proteins or 2) HFHS diet (Research Diets, D12331) comprising 58\% fat, $26 \%$ carbohydrates [ $17 \%$ sucrose], and $16 \%$ proteins) for six weeks. A female in the proestrus or estrus phase, determined by vaginal cytology, was mated overnight with a male. Females were checked daily for post-copulatory plugs, and a plug on the morning after mating was considered embryonic day (E) 0.5. Details of the number of animals used for 1) phenotypic testing and 2) for breeding to generate $F_{1}, F_{2}$, and $F_{3}$ in each group have previously been described ${ }^{15}$.

\section{Prenatal androgen exposure}

The CD and HFHS groups were in random order subdivided and subjected to daily injections subcutaneously (s.c.) in the interscapular area from E16.5 to E18.5 with $50 \mu \mathrm{l}$ of a 
solution containing: 1) a mixture of $5 \mu$ l benzyl benzoate (B6630; Sigma-Aldrich) and $45 \mu \mathrm{l}$ sesame oil (S3547; SigmaAldrich, St. Louis, Missouri, USA) i.e., vehicle, or 2) $250 \mu \mathrm{g}$ DHT ( $5 \alpha$ androstane-17 $\beta$-ol-3-one, A8380; Sigma-Aldrich, St. Louis, Missouri, USA) dissolved in a mixture of $5 \mu \mathrm{l}$ benzyl benzoate and $45 \mu \mathrm{l}$ sesame oil i.e., prenatal androgen exposure by DHT. In brief, prior to mating with male mice, $\mathrm{F}_{0}$ mothers were fed CD or HFHS-diet for 6 weeks ${ }^{15}$. During the embryonic day (E)16.5-E18.5 pregnant mice were injected subcutaneously with dihydrotestosterone (DHT), a non-aromatizable androgen, or sesame oil alone (vehicle) resulting in four experimental groups; MatCD+Vehicle (control), MatCD+DHT (androgenized), MatHFHS+Vehicle (obese), and MatHFHS+DHT (obese and androgenized). These are well-established models of prenatal androgen exposure and diet-induced obesity ${ }^{14,15} \cdot F_{1}$ female offspring were mated with unrelated healthy males fed $C D$ to generate $\mathrm{F}_{2}$ and thereafter to generate $\mathrm{F}_{3}$ female and male (siblings) offspring (female germline) (Fig. 2A). Furthermore, $F_{1}$ males were mated with unrelated health females fed $\mathrm{CD}$ to generate $\mathrm{mF}_{2}$ and $\mathrm{mF}_{3}$ males (male germline) (Fig. 2A and Table S4). Anxiety-like behavior was assessed in the elevated plus maze and in the open field at adult age in $F_{1}, F_{2}$ and $F_{3}$ female offspring and in their $\mathrm{F}_{2}$ and $\mathrm{F}_{3}$ male siblings $\left(\mathrm{fF}_{2}\right.$ and $\left.\mathrm{fF}_{3}\right)$, as well as in $\mathrm{F}_{1}, \mathrm{mF}_{2}$ and $\mathrm{mF}_{3}$ male offspring. Compromised embryonic development of second-generation $\left(\mathrm{F}_{2}\right)$ offspring in the obese and androgenized female lineage ${ }^{15}$ prevented us from investigating the transgenerational transmission of anxiety-like behavior in $\mathrm{F}_{3}$ offspring in the combined lineage.

\section{Mouse-breeding scheme and feeding paradigm to generate $F_{1}$ to $F_{3}$ generations}

All offspring, $F_{1}, F_{2}$, and $F_{3}$ were weaned at postnatal day 21 onto control diet: $C D+V e h, C D+D H T, H F H S+V e h$, and HFHS +DHT. To generate $F_{2}$, a subset of $F_{1}$ female and male offspring were mated with unrelated males and females fed the control diet, respectively, and a subset of $\mathrm{F}_{2}$ female and male offspring were mated with unrelated males and females, respectively, to generate $F_{3}$. Remaining $F_{1}, F_{2}$, and $F_{3}$ female and male offspring were subjected to phenotypic behavioral testing as described below.

\section{Prepubertal androgen exposure, in vitro fertilization, and offspring generation}

To generate the prepubertal PCOS-like mouse model, four week-old female mice were implanted subcutaneously with a 10-mm length pellet of crystalline $5 \alpha$-DHT (A83801G; Sigma-Aldrich, St. Louis, MO) and as control, a nonDHT containing pellet was inserted ${ }^{29}$. After 6 weeks of pellet implantation, $\mathrm{F}_{0}$ donors were subjected to phenotypic behavioral testing. Then these female mice (10-week of age) were superovulated and oocytes were collected and added to capacitated spermatozoa to perform in vitro fertilization (IVF) in the Karolinska Center for Transgene Technologies
(KCTT), Karolinska Institutet. Two-cell embryos were transferred into healthy surrogate mothers to generate $F_{1}$ female and male offspring. A subset of $F_{1}$ female and male offspring, respectively, underwent IVF to generate $F_{2}$ female and male offspring thus following both female and male germline. $F_{0}$ donors and remaining $F_{1}$ and $F_{2}$ female and male offspring were subjected to phenotypic behavioral testing as described below.

\section{Assessment of behavior}

All behavior experiments were performed at age of 16-17 weeks and during the light phase of the light-dark cycle, and mice were acclimatized in the testing room for 20-min before the test. In the EPM and OF tests, the mice were tracked automatically by an infrared digital camera using the EthoVision XT software (Noldus, Wageningen, the Netherlands). Innate fear/anxiety was assessed by using elevated plus maze as previously described ${ }^{14}$. Briefly, mice were placed into the middle of a four-armed maze facing an open arm and allowed to explore for 10-min. The number of arm entries and distance covered was tracked and analyzed by EthoVisionXT Software. We assessed anxiety-like behavior by calculating the percentage of time spent in the open and closed arms. For open field, the mouse was placed at the center of the arena and its movements were recorded for $10 \mathrm{~min}$. We evaluated anxiety-like behavior and general locomotor activity by calculating the percentage of time spent in the center and the total distance traveled, respectively. The OF test was performed 4 days after the EPM.

\section{Statistical analysis}

In the register-based cohort, children were followed from age 6, when they were eligible to receive a diagnosis of anxiety, to the date of diagnosis, death, emigration, age 18, or end of follow-up (December 31, 2013), whichever came first. The mean age and standard deviation at the end of follow-up were $11.2 \pm 3.6$ years. Associations between maternal PCOS and offspring anxiety were estimated as hazard ratios (HR) with $95 \%$ confidence intervals (CI) using stratified Cox regression models with attained age as the underlying time scale. In addition to the maternal matching criteria (maternal birth year and county of residence within the year of PCOS diagnosis), potential confounding variables were adjusted for including offspring sex and year of birth, maternal age at child's birth, maternal education, maternal region of birth, and maternal and paternal lifetime history of psychiatric disorders (adjusted Model). Robust standard errors were used to account for dependence between observations since several children from the same family were included in the study population. The analysis was conducted first for all offspring combined, then stratified by offspring sex. A sensitivity analysis including only the subset of the population with BMI data was conducted. 
In the mice experiments, data were assessed for normality and variance (Kolmogorov Smirnov). Group allocation during experiments was not blinded to the investigators. However, data analyses were repeated by two or more investigators. The sample size in the mice experiments was based on differences in AGD between the control and the androgenized lineage in our previous studies ${ }^{14,68}$. Nine animals per group were required to detect a mean difference in AGD of $40.6 \%$ with a standard deviation (SD) of 0.1, a significance level of 0.05 , and a power of 0.8 . All data are presented as mean \pm s.e.m, SD or as median and range. Differences between groups were determined by two-way ANOVA followed by Tukey's post hoc test or Bonferroni post hoc test, and by one-way ANOVA followed by Tukey's post hoc test or Bonferroni post hoc test. For the IVF experiment, we performed Student's $t$-test in normalized data. Differences were considered statistically significant at $P<0.05$. Statistical analyses for the register-based cohort study were performed using Stata statistical software version 15.1 (Stata Corps, Texas, USA), and in the animal studies by GraphPad Prism 8 (GraphPad Software Inc., CA, USA) and SPSS software v.26.0 (IBM, Armonk, NY, USA).

\section{Acknowledgements}

This work was funded by the Swedish Medical Research Council (Project No. 2014-2775, 2018-02435 E.S.V.; 2014-2870 Q.D.; 2018-02119 M.R.) and Swedish Initiative for Research on Microdata in the Social And Medical Sciences (SIMSAM) framework Grant No. 340-2013-5867; the Novo Nordisk Foundation (NNF17OC0026724, NNF18OC0033992 and NNF19OC0056647, E.S.V.); the SRP in Diabetes at Karolinska Institutet, E.S.V.; Adlerbertska Foundation (2019/86), E. S.V.; and Karolinska Institutet KID funding, E.S.V. and Q.D., Swedish Association of Medical Research (SSMF), Åke Wiberg Foundation, Q.D., and the Regional agreement on medical training and clinical research between Stockholm County Council and Karolinska Institutet, E.S.V., and Becas Chile-Conicyt (R.F.). The funders had no role in study design, data collection, and analysis, decision to publish, or preparation of the manuscript. Open Access funding provided by Karolinska Institute.

\section{Author details}

'Department of Physiology and Pharmacology, Karolinska Institutet, Stockholm, Sweden. 'Department of Medical Epidemiology and Biostatistics, Karolinska Institutet, Stockholm, Sweden. ${ }^{3}$ School of Medical Sciences, Örebro University, Örebro, Sweden. ${ }^{4}$ Department of Physiology, Sahlgrenska Academy, University of Gothenburg, Gothenburg, Sweden. ${ }^{5}$ School of Health Sciences, University of Skövde, Skövde, Sweden. ${ }^{6}$ Department of Medicine, Solna, Centre for Pharmacoepidemiology, Karolinska Institutet, Stockholm, Sweden

\section{Author contributions}

S.R. designed the mice study, performed the transgenerational behavioral phenotyping of the mice and collected data, analyzed the data of the targeted gene expression analyses of the amygdala, prepared the figures and was involved in writing the manuscript. M.M. assisted in the transgenerational behavioral phenotyping, design, and was involved in writing the manuscript. H.L. and R.F. assisted in the transgenerational behaviorally phenotyping and analyses. H.L., C.E.C., and M.A.R. provided data from the Swedish Nationwide register-based cohort study, analyzed these data, and was involved in writing the manuscript. A.B. and Q.D. were involved in the study design, interpretation of the results and assisted in the preparation of the manuscript. E.S.-V. designed the study, analyzed the data, prepared the figures and was involved in writing the manuscript.

\section{Data availability}

All data associated with this study are presented in the paper or supplementary materials.

\section{Conflict of interest}

Prof. Henrik Larsson has served as a speaker for Evolan and Shire and has received a research grant from Shire; all outside the scope of this submitted work. The other authors have no conflict of interest to declare.

\section{Publisher's note}

Springer Nature remains neutral with regard to jurisdictional claims in published maps and institutional affiliations.

Supplementary Information accompanies this paper at (https://doi.org/ 10.1038/s41398-020-01183-9).

Received: 14 September 2020 Revised: 30 November 2020 Accepted: 15 December 2020

Published online: 13 January 2021

\section{References}

1. Kataoka, J. et al. Prevalence of polycystic ovary syndrome in women with severe obesity-effects of a structured weight loss programme. Clin. Endocrinol. https://doi.org/10.1111/cen.14098 (2019).

2. March, W. A. et al. The prevalence of polycystic ovary syndrome in a community sample assessed under contrasting diagnostic criteria. Hum. Reprod. 25, 544-551 (2010).

3. Dumesic, D. A. et al. Scientific statement on the diagnostic criteria, epidemiology, pathophysiology, and molecular genetics of polycystic ovary syndrome. Endocr. Rev. 36, 487-525 (2015).

4. Cesta, C. E. et al. Polycystic ovary syndrome and psychiatric disorders: comorbidity and heritability in a nationwide Swedish cohort. Psychoneuroendocrinology 73, 196-203 (2016).

5. Pinola, P. et al. Androgen profile through life in women with polycystic ovary syndrome: A Nordic Multicenter Collaboration Study. J. Clin. Endocrinol. Metab. 100, 3400-3407 (2015).

6. Stener-Victorin, E. et al. Are there any sensitive and specific sex steroid markers for polycystic ovary syndrome? J. Clin. Endocrinol. Metab. 95, 810-819 (2010).

7. Cooney, L. G., Lee, I., Sammel, M. D. \& Dokras, A. High prevalence of moderate and severe depressive and anxiety symptoms in polycystic ovary syndrome: a systematic review and meta-analysis. Hum. Reprod. 32, 1075-1091 (2017).

8. Cesta, C. E. et al. Maternal polycystic ovary syndrome and risk of neuropsychiatric disorders in offspring: prenatal androgen exposure or genetic confounding? Psychol. Med. https://doi.org/10.1017/S0033291719000424 (2019).

9. Kosidou, K. et al. Maternal polycystic ovary syndrome and the risk of autism spectrum disorders in the offspring: a population-based nationwide study in Sweden. Mol. Psychiatry 21, 1441-1448 (2016).

10. Kosidou, K. et al. Maternal polycystic ovary syndrome and risk for attentiondeficit/hyperactivity disorder in the offspring. Biol. psychiatry 82, 651-659 (2017).

11. Robinson, S. L. et al. The associations of maternal polycystic ovary syndrome and hirsutism with behavioral problems in offspring. Fertil. Steril. 113, 435-443 (2020).

12. Chen, X., Kong, L., Piltonen, T. T., Gissler, M. \& Lavebratt, C. Association of polycystic ovary syndrome or anovulatory infertility with offspring psychiatric and mild neurodevelopmental disorders: a Finnish population-based cohort study. Hum. Reprod. 35, 2336-2347 (2020).

13. Hu, M. et al. Maternal testosterone exposure increases anxiety-like behavior and impacts the limbic system in the offspring. Proc. Natl Acad. Sci. USA 112, 14348-14353 (2015).

14. Manti, M. et al. Maternal androgen excess and obesity induce sexually dimorphic anxiety-like behavior in the offspring. Faseb J. https://doi.org/ 10.1096/fj.201701263RR (2018).

15. Risal, S. et al. Prenatal androgen exposure and transgenerational susceptibility to polycystic ovary syndrome. Nat. Med. 25, 1894-1904 (2019).

16. Chang, $\mathrm{H}$. et al. The protocadherin 17 gene affects cognition, personality, amygdala structure and function, synapse development and risk of major mood disorders. Mol. Psychiatry 23, 400-412 (2018).

17. Le-Niculescu, $\mathrm{H}$. et al. Convergent functional genomics of anxiety disorders: translational identification of genes, biomarkers, pathways and mechanisms. Transl. Psychiatry 1, e9 (2011) 
18. Wu, Y. E., Pan, L., Zuo, Y., Li, X. \& Hong, W. Detecting activated cell populations using single-cell RNA-Seq. Neuron 96, 313-329 (2017).

19. Vergult, S. et al. Genomic aberrations of the CACNA2D1 gene in three patients with epilepsy and intellectual disability. Eur. J. Hum. Genet. 23, 628-632 (2015)

20. Nordenankar, K., Bergfors, A. \& Wallen-Mackenzie, A. Targeted deletion of Vglut2 expression in the embryonal telencephalon promotes an anxiolytic phenotype of the adult mouse. Ups. J. Med. Sci. 120, 144-156 (2015).

21. Dunlop, B. W. \& Nemeroff, C. B. The role of dopamine in the pathophysiology of depression. Arch. Gen. Psychiatry 64, 327-337 (2007).

22. Miyata, S., Mori, Y. \& Tohyama, M. PRMT1 and Btg2 regulates neurite outgrowth of Neuro2a cells. Neurosci. Lett. 445, 162-165 (2008).

23. Passeri, D. et al. Btg2 enhances retinoic acid-induced differentiation by modulating histone $\mathrm{H} 4$ methylation and acetylation. Mol. Cell Biol. 26, 5023-5032 (2006).

24. French, C. A. \& Fisher, S. E. What can mice tell us about Foxp2 function? Curr. Opin. Neurobiol. 28, 72-79 (2014).

25. Rayman, J. B. et al. Genetic perturbation of TIA1 reveals a physiological role in fear memory. Cell Rep. 26, 2970-2983 (2019).

26. Coelho, J. E. et al. Overexpression of adenosine A2A receptors in rats: effects on depression, locomotion, and anxiety. Front. Psychiatry 5, 67 (2014).

27. Baekken, P. M., Skorpen, F., Stordal, E., Zwart, J. A. \& Hagen, K. Depression and anxiety in relation to catechol-O-methyltransferase Val158Met genotype in the general population: the Nord-Trondelag Health Study (HUNT). BMC Psychiatry 8, 48 (2008).

28. Pena, C. J. et al. Early life stress confers lifelong stress susceptibility in mice via ventral tegmental area OTX2. Science 356, 1185-1188 (2017).

29. Au-Xue, P. et al. A hyperandrogenic mouse model to study polycystic ovary syndrome. JoVE https://doi.org/10.3791/58379 (2018).

30. Martínez, D. et al. In utero undernutrition in male mice programs liver lipid metabolism in the second-generation offspring involving altered Lxra DNA methylation. Cell Metab. 19, 941-951 (2014).

31. Chen, Q. et al. Sperm tsRNAs contribute to intergenerational inheritance of an acquired metabolic disorder. Science 351, 397-400 (2016).

32. Arnold, A. P. The organizational-activational hypothesis as the foundation for a unified theory of sexual differentiation of all mammalian tissues. Horm. Behav. 55, 570-578 (2009).

33. Auyeung, B. et al. Fetal testosterone predicts sexually differentiated childhood behavior in girls and in boys. Psychol. Sci. 20, 144-148 (2009).

34. Glintborg, D. et al. Testosterone levels in third trimester in polycystic ovary syndrome: odense child cohort. J. Clin. Endocrinol. Metab. 103, 3819-3827 (2018).

35. Kollmann, M. et al. Androgen and anti-Mullerian hormone concentrations at term in newborns and their mothers with and without polycystic ovary syndrome. J. Clin. Med. https://doi.org/10.3390/jcm8111817 (2019).

36. Falbo, A. et al. Changes in androgens and insulin sensitivity indexes throughout pregnancy in women with polycystic ovary syndrome (PCOS): relationships with adverse outcomes. J. Ovarian Res. 3, 23 (2010).

37. Barry, J. A. et al. Umbilical vein testosterone in female infants born to mothers with polycystic ovary syndrome is elevated to male levels. J. Obstet. Gynaecol. 30, 444-446 (2010).

38. Anderson, $\mathrm{H}$. et al. Infants of women with polycystic ovary syndrome have lower cord blood androstenedione and estradiol levels. J. Clin. Endocrinol. Metab. 95, 2180-2186 (2010).

39. Maliqueo, M. et al. Placental steroidogenesis in pregnant women with polycystic ovary syndrome. Eur. J. Obstet. Gynecol. Reprod. Biol. 166, 151-155 (2013).

40. Sir-Petermann, T. et al. Maternal serum androgens in pregnant women with polycystic ovarian syndrome: possible implications in prenatal androgenization. Hum. Reprod. 17, 2573-2579 (2002).

41. Morris, J. A., Jordan, C. L., King, Z. A., Northcutt, K. V. \& Breedlove, S. M. Sexual dimorphism and steroid responsiveness of the posterodorsal medial amygdala in adult mice. Brain Res. 1190, 115-121 (2008).

42. Spiers, H. et al. Methylomic trajectories across human fetal brain development. Genome Res. 25, 338-352 (2015).

43. Li, M. et al. Integrative functional genomic analysis of human brain development and neuropsychiatric risks. Science 362, eaat7615 (2018).

44. Lane, M., Zander-Fox, D. L., Robker, R. L. \& McPherson, N. O. Peri-conception parental obesity, reproductive health, and transgenerational impacts. Trends Endocrinol. Metab. 26, 84-90 (2015).
45. Bale, T. L. Epigenetic and transgenerational reprogramming of brain development. Nat. Rev. Neurosci. 16, 332-344 (2015).

46. Winther, G., Elfving, B., Muller, H. K., Lund, S. \& Wegener, G. Maternal high-fat diet programs offspring emotional behavior in adulthood. Neuroscience $\mathbf{3 8 8}$ 87-101 (2018).

47. Lin, C., Shao, B., Huang, H., Zhou, Y. \& Lin, Y. Maternal high fat diet programs stress-induced behavioral disorder in adult offspring. Physiol. Behav. 152, 119-127 (2015).

48. Grundwald, N. J. \& Brunton, P. J. Prenatal stress programs neuroendocrine stress responses and affective behaviors in second generation rats in a sex-dependent manner. Psychoneuroendocrinology 62, 204-216 (2015).

49. Constantinof, A., Moisiadis, V. G. \& Matthews, S. G. Programming of stress pathways: a transgenerational perspective. J. Steroid Biochem. Mol. Biol. 160, 175-180 (2016).

50. lqbal, M., Moisiadis, V. G., Kostaki, A. \& Matthews, S. G. Transgenerational effects of prenatal synthetic glucocorticoids on hypothalamic-pituitary-adrenal function. Endocrinology 153, 3295-3307 (2012).

51. Rando, O. J. Daddy issues: paternal effects on phenotype. Cell 151, 702-708 (2012).

52. McEwen, B. S. Stress, sex, and neural adaptation to a changing environment: mechanisms of neuronal remodeling. Ann. N. Y. Acad. Sci. 1204, E38-E59 (2010).

53. Wang, T. et al. CAMK2N1 inhibits prostate cancer progression through androgen receptor-dependent signaling. Oncotarget 5, 10293-10306 (2014).

54. Romanuik, T. L. et al. Identification of novel androgen-responsive genes by sequencing of LongSAGE libraries. BMC Genom. 10, 476 (2009).

55. Nguyen, R. L., Medvedeva, Y. V., Ayyagari, T. E., Schmunk, G. \& Gargus, J. J. Intracellular calcium dysregulation in autism spectrum disorder: an analysis of converging organelle signaling pathways. Biochim Biophys. Acta 1865, 1718-1732 (2018).

56. Hettema, J. M. et al. Catechol-O-methyltransferase contributes to genetic susceptibility shared among anxiety spectrum phenotypes. Biol. Psychiatry 64, 302-310 (2008).

57. Koh, M. J., Kang, J. I., Namkoong, K., Lee, S. Y. \& Kim, S. J. Association between the catechol-O-methyltransferase (COMT) Val(1)(5)(8)Met polymorphism and alexithymia in patients with obsessive-compulsive disorder. Yonsei Med. J. $\mathbf{5 7}$ 721-727 (2016).

58. Annerbrink, K. et al. Catechol O-methyltransferase val158-met polymorphism is associated with abdominal obesity and blood pressure in men. Metabolism 57, 708-711 (2008).

59. Rincel, M. et al. Multi-hit early life adversity affects gut microbiota, brain and behavior in a sex-dependent manner. Brain Behav. Immun. 80, 179-192 (2019).

60. Kurumaji, A., Ito, T., Ishii, S. \& Nishikawa, T. Effects of FG7142 and immobilization stress on the gene expression in the neocortex of mice. Neurosci. Res. 62 , 155-159 (2008).

61. de Haas, T. et al. OTX1 and OTX2 expression correlates with the clinicopathologic classification of medulloblastomas. J. Neuropathol. Exp. Neurol. 65, 176-186 (2006).

62. Acampora, D. et al. Epilepsy and brain abnormalities in mice lacking the Otx 1 gene. Nat. Genet. 14, 218-222 (1996).

63. Janak, P. H. \& Tye, K. M. From circuits to behaviour in the amygdala. Nature 517, 284-292 (2015)

64. McDonald, A. J. Cortical pathways to the mammalian amygdala. Prog. Neurobiol. 55, 257-332 (1998).

65. Bitsko, R. H. et al. Epidemiology and impact of health care provider-diagnosed anxiety and depression among US children. J. Dev. Behav. Pediatr. 39, 395-403 (2018).

66. Lijster, J. M. et al. The age of onset of anxiety disorders. Can. J. Psychiatry Rev 62, 237-246 (2017).

67. The Rotterdam ESHRE/ASRM-Sponsored PCOS Consensus Workshop Group Revised 2003 consensus on diagnostic criteria and long-term health risks related to polycystic ovary syndrome. Fertil. Steril. 81, 19-25 (2004).

68. Manti, M. et al. Maternal androgen excess induces cardiac hypertrophy and left ventricular dysfunction in female mice offspring. Cardiovasc. Res. https://doi.org/10.1093/cvr/cvz180 (2019). 